\title{
On the genus of non-zero component union graphs of vector spaces
}

\author{
Gnanappirakasam Kalaimurugan ${ }^{1}$ (D), Singaravelu Gopinath ${ }^{1}$ (D), Thirugnanam \\ Tamizh Chelvam*2 (D) \\ ${ }^{1}$ Department of Mathematics, Thiruvalluvar University, Vellore 632115, Tamil Nadu, India \\ ${ }^{2}$ Department of Mathematics, Manonmaniam Sundaranar University, Tirunelveli 627012, Tamil Nadu, \\ India
}

\begin{abstract}
Let $\mathbb{V}$ be an $n$-dimensional vector space over the field $\mathbb{F}$ with a basis $\mathfrak{B}=\left\{\alpha_{1}, \ldots, \alpha_{n}\right\}$. For a non-zero vector $v \in \mathbb{V} \backslash\{0\}$, the skeleton of $v$ with respect to the basis $\mathbb{B}$ is defined as $S_{\mathfrak{B}}(v)=\left\{\alpha_{i}: v=\sum_{i=1}^{n} a_{i} \alpha_{i}, a_{i} \neq 0\right\}$. The non-zero component union graph $\Gamma\left(\mathbb{V}_{\mathfrak{B}}\right)$ of $\mathbb{V}$ with respect to $\mathfrak{B}$ is the simple graph with vertex set $V=\mathbb{V} \backslash\{0\}$ and two distinct non-zero vectors $u, v \in V$ are adjacent if and only if $S_{\mathfrak{B}}(u) \cup S_{\mathfrak{B}}(v)=\mathfrak{B}$. First, we obtain some graph theoretical properties of $\Gamma\left(\mathbb{V}_{\mathfrak{B}}\right)$. Further, we characterize all finite dimensional vector spaces $\mathbb{V}$ for which $\Gamma\left(\mathbb{V}_{\mathfrak{B}}\right)$ has genus either 0 or 1 or 2 . In the last part of the paper, we characterize all finite dimensional vector spaces $\mathbb{V}$ for which the cross cap of $\Gamma\left(\mathbb{V}_{\mathfrak{B}}\right)$ is 1 .
\end{abstract}

Mathematics Subject Classification (2020). 05C10, 05C25, 05C75

Keywords. vector space, finite dimensional, non-zero component union graph, genus

\section{Introduction}

The investigation of mathematical structures utilizing the properties of graphs has become an energizing exploration theme over the most recent couple of decades, prompting numerous intriguing outcomes and questions. The study of algebraic properties by using the discrete structure of graphs was instituted by Beck[2] to introduce and study about the zero-divisor graph of a commutative ring with unity. Also different type of graphs from algebraic structures are defined and studied in $[1,6]$. Das $[4,5]$ defined and studied about various graphs from vector spaces. Further studies of graphs from vector spaces were also appeared in $[5,12,13]$. More specifically, Das has introduced and studied two graphs viz., non-zero component graph [5] and non-zero component union graph[4] of vector spaces. Das[4] studied about the domination number, the clique number and the chromatic number of non-zero component union graph of finite-dimensional vector spaces. Recent years, graph embeddings in a surface is an interesting area of research and for some of the studies one can refer[10,14]. Also the crosscap of are studied in $[8,11]$. Tamizh Chelvam and

*Corresponding Author.

Email addresses: kalaimurugan@gmail.com(G. Kalaimurugan), gopinathmathematics@gmail.com(S. Gopinath), tamche59@gmail.com (T. Tamizh Chelvam)

Received: 18.06.2020; Accepted: 08.06.2021 
Prabha Ananthi[12] studied about genus properties of non-zero component graph of finite dimensional vector spaces. In this paper, we are interested about the genus of the non-zero component union graph of finite-dimensional vector spaces.

\section{Preliminaries}

Let $\mathbb{V}$ be a finite dimensional vector space over the field $\mathbb{F}$ with $\mathfrak{B}=\left\{\alpha_{1}, \alpha_{2}, \ldots, \alpha_{n}\right\}$ as a basis. Throughout this paper, $\mathbb{V}$ is a finite dimensional vector space with dimension $n$ over the finite field $\mathbb{F}$ with $q$ elements. Any vector $v \in \mathbb{V}$ can be expressed uniquely as a linear combination $v=a_{1} \alpha_{1}+a_{2} \alpha_{2}+\ldots+a_{n} \alpha_{n}$ where $a_{i} \in \mathbb{F}$ and the same is denoted by $v=\left(a_{1}, a_{2}, \ldots, a_{n}\right)$. The skeleton of $v \in \mathbb{V}^{*}$ with respect to $\mathfrak{B}$ is defined as $S_{\mathfrak{B}}(v)=\left\{\alpha_{i}: a_{i} \neq 0, i=1,2, \ldots, n\right\}$. The non-zero component union graph $\Gamma\left(\mathbb{V}_{\mathfrak{B}}\right)$ of $\mathbb{V}$ with respect to $\mathfrak{B}$ is the simple graph with vertex set $V=\mathbb{V} \backslash\{0\}$ and two distinct non-zero vectors $u, v \in V$ are adjacent if and only if $S_{\mathfrak{B}}(u) \cup S_{\mathfrak{B}}(v)=\mathfrak{B}$.

Now, let us recall basic definitions and notations about graphs. By a graph $G=(V, E)$, we mean a simple graph with non-empty vertex set $V$ and edge set $E$. The number of elements in $V$ is called order of $G$ and the number of elements in $E$ is called the size of $G$. A graph $G$ is said to be complete if every pair of distinct vertices is adjacent in $G$. We denote the complete graph of order $n$ by $K_{n}$. A graph $G$ is bipartite if the vertex $V$ can be partitioned into two disjoint subsets with no pair of vertices in one subset is adjacent. A star graph is a bipartite graph with any one of the partition containing a single vertex and the same is called as the center of the star. A graph $G$ is connected if there exists a path between every pair of distinct vertices in $G$. The degree of the vertex $v \in V$, denoted by $d(v)$, is the number of edges in $G$ which are incident with $v$. A graph $G$ is said to be r-regular if the degree of all the vertices in $G$ is $r$. The diameter of a connected graph is supremum of shortest distances between pairs of distinct vertices in $G$ and is denoted by $\operatorname{diam}(G)$. The girth of $G$ is defined as length of the shortest cycle in $G$ and is denoted by $\operatorname{gr}(G)$. We take $\operatorname{gr}(G)=\infty$ if $G$ contains no cycles. For undefined terms in graph theory, we refer[3]

A graph $G$ is said to be embedded in a surface $S$ if $G$ can be drawn in $S$ such that edges intersect only at vertices of $G$. Intuitively, $G$ is embedded in a surface if it can be drawn in the surface so that its edges intersect only at their common vertices. The genus of a graph $G$ is denoted by $g(G)$, is the minimum integer $n$ such that the graph can be embedded in $S_{n}$, where $S_{n}$ denotes the sphere with $n$ handles. A planar graph is a graph that can be embedded in the plane and the genus of planar graphs is zero. A graph $G$ is called toroidal if genus of $G$ is one. Let $\bar{S}_{k}$ denote the sphere with $k$ crosscaps, where $k$ is a non-negative integer, that is, $\bar{S}_{k}$ is a non-oriented surface with $k$ crosscaps. The crosscap of a graph $G$, denoted $\bar{g}(G)$, is the minimal integer $n$ such that the graph $G$ can be embedded in $\bar{S}_{n}$. We say a graph $G$ is planar if $\bar{g}(G)=0$, and projective if $\bar{g}(G)=1$. It is easy to see that $\bar{g}(H) \leq \bar{g}(G)$ for a subgraph $H$ of $G$.

We list out certain existing results which will be referred in this paper.

Theorem 2.1. ([4, Theorem 4.2]) Let $\mathbb{V}$ be an $n$-dimensional vector space over a finite field $\mathbb{F}$ with q elements. Then $\Gamma\left(\mathbb{V}_{\mathfrak{B}}\right)$ is complete if and only if $\mathbb{V}$ is one-dimensional or $\mathbb{V}$ is two-dimensional and $|\mathbb{F}|=2$.

Theorem 2.2. ([4, Theorem 6.3]) Let $\mathbb{V}$ be an $n$-dimensional vector space over a finite field $\mathbb{F}$ with $q$ elements. Then the clique number and chromatic number of $\Gamma\left(\mathbb{V}_{\mathfrak{B}}\right)$ are both equal to $n+(q-1)^{n}$. i.e., $\Gamma\left(\mathbb{V}_{\mathfrak{B}}\right)$ is weakly perfect.

Theorem 2.3. ([4, Theorem 6.1]) Let $\mathbb{V}$ be an $n$-dimensional vector space over a finite field $\mathbb{F}$ with $q$ elements. Let $\Gamma\left(\mathbb{V}_{\mathfrak{B}}\right)$ be the non-zero component union graph of $\mathbb{V}$ with 
respect to the basis $\mathfrak{B}=\left\{\alpha_{1}, \alpha_{2}, \ldots, \alpha_{n}\right\}$. Let $v=c_{1} \alpha_{i_{1}}+c_{2} \alpha_{i_{2}}+\ldots+c_{k} \alpha_{i_{k}}$ be a vertex in $\Gamma\left(\mathbb{V}_{\mathfrak{B}}\right)$ with $c_{1}, c_{2}, \ldots, c_{k} \neq 0$. Then $\operatorname{deg}(v)= \begin{cases}(q-1)^{n-k} q^{k} & \text { if } 1 \leq k \leq n \\ q^{n}-2 & \text { if } k=n .\end{cases}$

Theorem 2.4. ([4, Theorem 6.2]) Let $\mathbb{V}$ be an $n$-dimensional vector space over a finite field $\mathbb{F}$ with $q$ elements. Then the order of $\Gamma\left(\mathbb{V}_{\mathfrak{B}}\right)$ is $q^{n}-1$ and the size $m$ of $\Gamma\left(\mathbb{V}_{\mathfrak{B}}\right)$ is $\frac{(q-1)^{n}\left[(q+1)^{n}-3\right]}{2}$.

Theorem 2.5. ([4, Corollary 6.1])Let $\mathbb{V}$ be an $n$-dimensional vector space over a finite field $\mathbb{F}$ with $q$ elements. The maximum and minimum degree of $\Gamma\left(\mathbb{V}_{\mathfrak{B}}\right)$ is $\Delta=q^{n}-2$ and $\delta=q(q-1)^{n-1}$.

Theorem 2.6. ([4, Theorem 7.1]) $I=\left\{\alpha \in \Gamma\left(\mathbb{V}_{\mathfrak{B}}\right): S(\alpha) \subseteq\left\{\alpha_{1}, \alpha_{2}, \ldots, \alpha_{n-1}\right\}\right\}$ is a maximal independent set in $\Gamma\left(\mathbb{V}_{\mathfrak{B}}\right)$. Moreover if $\mathbb{F}$ is a finite field with $q$ elements, $|I|=q^{n-1}-1$.

Lemma 2.7. ([15, Theorem 6.38]) $g\left(K_{n}\right)=\left\lceil\frac{(n-3)(n-4)}{12}\right\rceil$ if $n \geq 3$. In particular $g\left(K_{n}\right)=1$ if $n=5,6,7$.

Lemma 2.8. ([15, Corollary 6.12]) If $G$ is a finite connected graph with $n$ vertices, $m$ edges, and genus $g$, then $n-m+f=2-2 g$, where $f$ is the number of faces created when $G$ is minimally embedded on a surface of genus $g$.

Theorem 2.9. ([7, pp. 115]) Let $m, n$ be integers and for a real number $x,\lceil x\rceil$ is the least integer that is greater than or equal to $x$. Then

(1) $\bar{g}\left(K_{n}\right)= \begin{cases}\left\lceil\frac{(n-3)(n-4)}{6}\right\rceil & \text { if } n \geq 3 \text { and } n \neq 7 \\ 3 & \text { if } n=7 .\end{cases}$

(2) $\bar{g}\left(K_{m, n}\right)=\left\lceil\frac{(m-2)(n-2)}{2}\right\rceil$ where $m, n \geq 2$.

Proposition 2.10. [15, Theorem 5.14] Let $\varphi: G \rightarrow N_{k}$ be a 2-cell embedding of a connected graph $G$ to the non-orientable surface $N_{k}$. Then $v-e+f=2-k$, where $v, e$ and $f$ are the numbers of vertices, edges, and faces that $\varphi(G)$ has respectively, and $k$ is the crosscap of $N_{k}$.

Theorem 2.11. ([9, Theorem 1]) A graph $G$ is outerplanar if and only if it contains no subgraph homeomorphic to $K_{4}$ or $K_{2,3}$ except $K_{4}-x$, where $x$ denotes an edge of $K_{4}$.

\section{Properties of $\Gamma\left(\mathbb{V}_{\mathfrak{B}}\right)$}

In this section, we characterize all finite dimensional vector spaces whose non-zero component union graph is unicyclic or claw-free. Recall that a graph $G$ is said to be unicyclic, if it contains a unique cycle. A graph $G$ is said to be claw-free if $G$ does not contain $K_{1,3}$ as an induced subgraph of $G$.

Theorem 3.1. Let $n \geq 1$ and $q \geq 2$ be integers. Let $\mathbb{V}$ be an $n$ dimensional vector space over the field $\mathbb{F}$ with $q$ elements. Then $\Gamma\left(\mathbb{V}_{\mathfrak{B}}\right)$ is unicyclic if and only if either $(n=1$ and $q=4)$ or $(n=2$ and $q=2)$.

Proof. Assume that $\Gamma\left(\mathbb{V}_{\mathfrak{B}}\right)$ is unicyclic. First we claim that $n \leq 2$ and $q \leq 4$.

Suppose $n \geq 3$. Note that the vertices $u_{1}=(1,1, \ldots, 1), u_{2}=(1,1,0,1,1, \ldots, 1), u_{3}=$ $(0,0,1,1, \ldots, 1), u_{4}=(1,0,1,1, \ldots, 1)$ and $u_{5}=(0,1,1, \ldots, 1)$ form two distinct cycles $u_{1}-u_{2}-u_{3}-u_{1}$ and $u_{1}-u_{4}-u_{5}-u_{1}$ in $\Gamma\left(\mathbb{V}_{\mathfrak{B}}\right)$, which is a contradiction. Hence $n \leq 2$.

Suppose $q \geq 5$. Then $\mathbb{F}$ contains non-zero elements $a_{1}, a_{2}, a_{3}$ and $a_{i} \neq 1$ for $1 \leq$ $i \leq 3$. Now, the vertices $v_{1}=(1,1, \ldots, 1), v_{2}=\left(a_{1}, 1,1, \ldots, 1\right), v_{3}=\left(a_{2}, 1, \ldots, 1\right), v_{4}=$ $\left(a_{3}, 1, \ldots, 1\right)$ and $v_{5}=(0,1,1, \ldots, 1)$ form two distinct cycles $v_{1}-v_{2}-v_{3}-v_{1}$ and $v_{1}-v_{4}-v_{5}-v_{1}$ which is a contradiction. 
Thus we have $n \leq 2$ and $q \leq 4$.

Case 1. Let $n=1$. If $q$ is either 2 or 3 , then by Theorem $2.1, \Gamma\left(\mathbb{V}_{\mathfrak{B}}\right)$ is isomorphic to either $K_{1}$ or $K_{2}$ respectively, it contradicts to the fact that $\Gamma\left(\mathbb{V}_{\mathfrak{B}}\right)$ contains a unique cycle. This implies that $n=1$ and $q=4$.

Case 2. Let $n=2$. Suppose that $q>2$. Then $\mathbb{F}$ contains an element $a_{1}$ with $a_{1} \neq 0$ and $a_{1} \neq 1$. Now, the vertices $u_{1}=(1,1), u_{2}=(0,1), u_{3}=(1,0), u_{4}=\left(a_{1}, a_{1}\right), u_{5}=\left(a_{1}, 0\right)$ and $u_{6}=\left(0, a_{1}\right)$ of $\Gamma\left(\mathbb{V}_{\mathfrak{B}}\right)$ form two distinct cycles $u_{1}-u_{2}-u_{3}-u_{1}$ and $u_{4}-u_{5}-u_{6}-u_{4}$, a contradiction. Hence $n=2$ and $q=2$.

Conversely, assume that $(n=1$ and $q=4)$ or $(n=2$ and $q=2)$. In both the cases, by Theorems 2.1 and $2.4, \Gamma\left(\mathbb{V}_{\mathfrak{B}}\right) \cong K_{3}$ and so it is unicyclic.

Theorem 3.2. Let $n \geq 1$ and $q \geq 2$ be integers. Let $\mathbb{V}$ be an $n$-dimensional vector space over the field $\mathbb{F}$ with $q$ elements. Then $\Gamma\left(\mathbb{V}_{\mathfrak{B}}\right)$ is claw-free if and only if either $(n=1)$ or $(n=2$ and $q \leq 3)$.

Proof. Assume that $\Gamma\left(\mathbb{V}_{\mathfrak{B}}\right)$ is claw-free. Suppose $n \geq 3$. Then the vertices $\{(1,1, \ldots, 1)$, $(1,0,0, \ldots, 0),(0,1,0,0, \ldots, 0),(0,0,1,0,0, \ldots, 0)\}$ induce $K_{1,3}$ as an induced subgraph of $\Gamma\left(\mathbb{V}_{\mathfrak{B}}\right)$, which is a contradiction. Hence $n \leq 2$.

If $n=1$, then there is nothing to prove. If $n=2$ and $q \geq 4$, then $\mathbb{F}$ contains $a_{1}$ and $a_{2}$ with $a_{1}, a_{2} \neq 0$ and $a_{1}, a_{2} \neq 1$. From this, the vertices $u_{1}=(1,1), u_{2}=(0,1), u_{3}=\left(0, a_{1}\right)$ and $u_{4}=\left(0, a_{2}\right)$ form $K_{1,3}$ as an induced subgraph of $\Gamma\left(\mathbb{V}_{\mathfrak{B}}\right)$ with center at $u_{1}$, which is a contradiction. Hence $q \leq 3$.

Thus we have, either $(n=1)$ or $(n=2$ and $q \leq 3)$.

Conversely, assume that either $(n=1)$ or $(n=2$ and $q \leq 3)$. If $n=1$, by Theorem 2.1, $\Gamma\left(\mathbb{V}_{\mathfrak{B}}\right)$ is complete and hence claw free. If $n=2$ and $q=2$, then by Theorems 2.1 and 2.4, $\Gamma\left(\mathbb{V}_{\mathfrak{B}}\right) \cong K_{3}$ and hence claw-free. If $n=2$ and $q=3$, then the graph $\Gamma\left(\mathbb{V}_{\mathfrak{B}}\right)$ is given in Fig. 1. One can check that the graph $\Gamma\left(\mathbb{V}_{\mathfrak{B}}\right)$ given in Fig. 1 is claw-free.

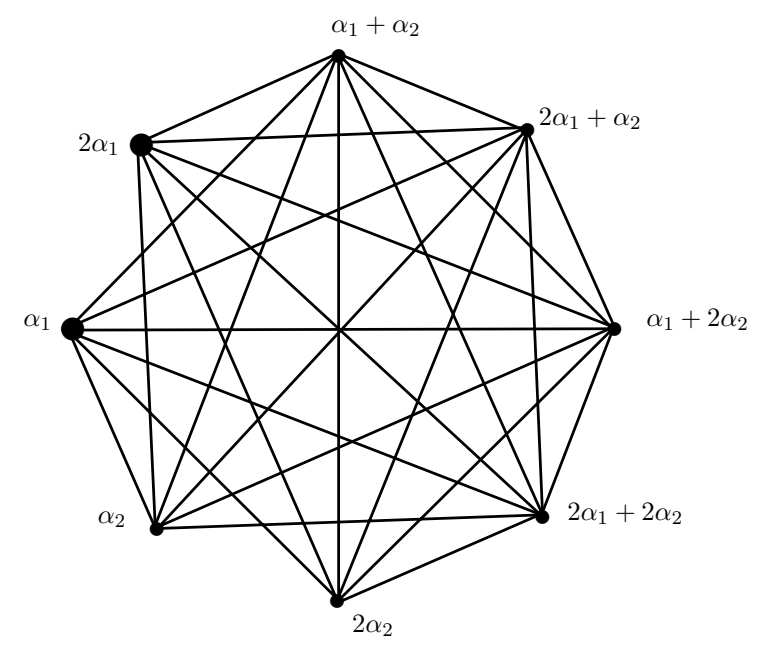

Fig. 1: $\Gamma\left(\mathbb{V}_{\mathfrak{B}}\right)$ where $\operatorname{dim}(\mathbb{V})=2$ and $\mathbb{F}=\mathbb{Z}_{3}$.

\section{Genus of $\Gamma\left(\mathbb{V}_{\mathfrak{B}}\right)$}

In this section, we characterize all finite dimensional vector spaces $\mathbb{V}$ over a finite field $\mathbb{F}$ whose $\Gamma\left(\mathbb{V}_{\mathfrak{B}}\right)$ is planar.

Theorem 4.1. Let $n \geq 1$ and $q \geq 2$ be integers. Let $\mathbb{V}$ be an $n$-dimensional vector space over the field $\mathbb{F}$ with $q$ elements. Then $\Gamma\left(\mathbb{V}_{\mathfrak{B}}\right)$ is planar if and only if either $(n=1$ and $q \leq 5)$ or $(n=2$ and $q=2)$ or $(n=3$ and $q=2)$.

Proof. Let us assume that $\Gamma\left(\mathbb{V}_{\mathfrak{B}}\right)$ is planar. First we claim that $n \leq 3$ and $q \leq 5$. 
Suppose $n \geq 4$. Let $u_{1}=(1,1, \ldots, 1), u_{2}=(0,1,1, \ldots, 1), u_{3}=(1,0,1,1, \ldots, 1), u_{4}=$ $(1,1,0,1,1, \ldots, 1)$ and $u_{5}=(1,1,1,0,1,1, \ldots, 1)$. Then the induced subgraph of $\Gamma\left(\mathbb{V}_{\mathfrak{B}}\right)$ induced by $\Omega=\left\{u_{1}, u_{2}, u_{3}, u_{4}, u_{5}\right\}$ is $K_{5}$, which is a contradiction to $\Gamma\left(\mathbb{V}_{\mathfrak{B}}\right)$ is planar. Hence $n \leq 3$.

Suppose $q \geq 6$. Then $\mathbb{F}$ contains $0,1 \neq a_{i}$ for $1 \leq i \leq 4$. Consider the vertices $u_{1}=(1,1, \ldots, 1), u_{2}=\left(a_{1}, 1,1, \ldots, 1\right), u_{3}=\left(a_{2}, 1,1, \ldots, 1\right), u_{4}=\left(a_{3}, 1,1, \ldots, 1\right)$ and $u_{5}=$ $\left(a_{4}, 1,1, \ldots, 1\right)$. Then the induced subgraph of $\Gamma\left(\mathbb{V}_{\mathfrak{B}}\right)$ induced by $\Omega=\left\{u_{1}, u_{2}, u_{3}, u_{4}, u_{5}\right\}$ is $K_{5}$, which is a contradiction to $\Gamma\left(\mathbb{V}_{\mathfrak{B}}\right)$ is planar. Hence $q \leq 5$.

Thus we have, $n \leq 3$ and $q \leq 5$.

Case 1. Suppose $n=3$ and $q \geq 3$. Then $\mathbb{F}$ contains $0,1 \neq a_{1}$. Let $u_{1}=(1,1,1), u_{2}=$ $\left(a_{1}, a_{1}, a_{1}\right), u_{3}=\left(1,1, a_{1}\right), u_{4}=\left(1, a_{1}, 1\right), u_{5}=\left(a_{1}, 1,1\right)$ and $\Omega=\left\{u_{1}, u_{2}, u_{3}, u_{4}, u_{5}\right\}$. Note that the induced subgraph of $\Gamma\left(\mathbb{V}_{\mathfrak{B}}\right)$ induced by $\Omega$ is $K_{5}$, which is a contradiction. Hence $q=2$.

Case 2. Suppose $n=2$ and $q \geq 3$. Then $\mathbb{F}$ contains $0,1 \neq a_{1}$. Let $u_{1}=(1,1), u_{2}=$ $\left(a_{1}, 1\right), u_{3}=\left(1, a_{1}\right), u_{4}=\left(a_{1}, a_{1}\right), u_{5}=(0,1)$ and $\Omega=\left\{u_{1}, u_{2}, u_{3}, u_{4}, u_{5}\right\}$. Note that $\langle\Omega\rangle=K_{5}$ is an induced subgraph of $\Gamma\left(\mathbb{V}_{\mathfrak{B}}\right)$, which is a contradiction. Hence $q=2$.

Case 3. Suppose $n=1$ and $q \geq 6$. By Theorem 2.1, $\Gamma\left(\mathbb{V}_{\mathfrak{B}}\right)$ is complete. Since $q \geq 6, K_{5}$ is a subgraph in $\Gamma\left(\mathbb{V}_{\mathfrak{B}}\right)$, which is a contradiction to the fact that $\Gamma\left(\mathbb{V}_{\mathfrak{B}}\right)$ is planar. Hence $q \leq 5$.

Conversely, if $n=1$ and $q \leq 5$. By Theorems 2.1 and $2.7, \Gamma\left(\mathbb{V}_{\mathfrak{B}}\right)$ is the complete graph $K_{t}$ for $t \leq 4$. Thus $\Gamma\left(\mathbb{V}_{\mathfrak{B}}\right)$ is planar.

If $n=2$ and $q=2$, then $\mathbb{V} \cong \mathbb{Z}_{2} \times \mathbb{Z}_{2}$ and $\mathbb{F}=\mathbb{Z}_{2}$. In this case, $\Gamma\left(\mathbb{V}_{\mathfrak{B}}\right)=C_{3}$, which is trivially a planar graph.

If $n=3$ and $q=2$, then $\mathbb{V} \cong \mathbb{Z}_{2} \times \mathbb{Z}_{2} \times \mathbb{Z}_{2}$ and $\mathbb{F}=\mathbb{Z}_{2}$. A planar embedding of $\Gamma\left(\mathbb{V}_{\mathfrak{B}}\right)$ is given in Fig. 2 and hence in this case also $\Gamma\left(\mathbb{V}_{\mathfrak{B}}\right)$ is planar.

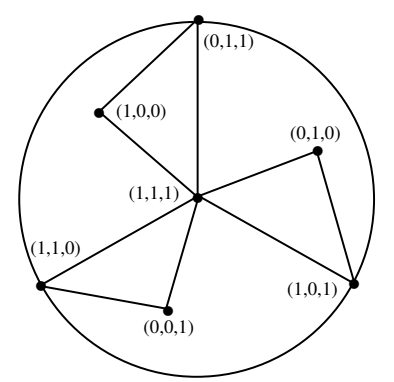

Fig. 2: $\Gamma\left(\mathbb{Z}_{2} \times \mathbb{Z}_{2} \times \mathbb{Z}_{2}\right)$ over $\mathbb{F}=\mathbb{Z}_{2}$

In the following theorems, we characterize all finite dimensional vector spaces $\mathbb{V}$ over finite field $\mathbb{F}$ for which $\Gamma\left(\mathbb{V}_{\mathfrak{B}}\right)$ is toroidal.

Lemma 4.2. For the graph $H$ given in Fig. $3, g(H) \geq 2$. 


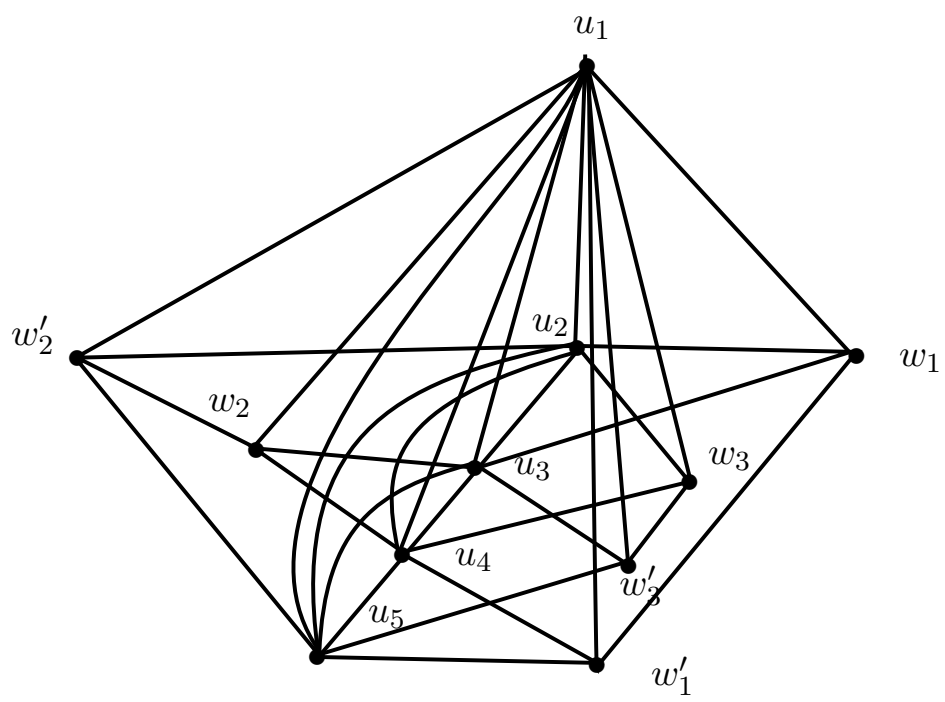

Fig. 3: Graph $H$

Proof. Note that the induced subgraph $H^{\prime}=\left\langle\left\{u_{1}, u_{2}, u_{3}, u_{4}, u_{5}\right\}\right\rangle$ of $\Gamma\left(\mathbb{V}_{\mathfrak{B}}\right)$ is $K_{5}$. By Lemma 2.7, $g\left(H^{\prime}\right)=1$. Since $H^{\prime}$ contains 5 vertices and 10 edges, by Theorem 2.8, any embedding of $H^{\prime}$ in $S_{1}$ has 5 faces namely $f_{1}^{\prime}, f_{2}^{\prime}, \ldots, f_{5}^{\prime}$. Let $f_{k}$ be the number of $k$-gons in $H^{\prime}$. Then $20=2 e=\sum_{k>3} k f_{k} \geq 3 f_{3}+4 f_{4}+5 f_{5}$.

Note that, $\left\{w_{1}, w_{1}^{\prime}\right\},\left\{w_{2}, w_{2}^{\prime}\right\}$ and $\left\{w_{3}, w_{3}^{\prime}\right\}$ are pair of adjacent vertices with one common neighbourhood $u_{1}$. Also, each pair of vertices is adjacent with two distinct vertices in $\left\{u_{2}, u_{3}, u_{4}, u_{5}\right\}$. Now, to insert one pair from $\left\{\left(w_{j}, w_{j}^{\prime}\right): j=1,2,3\right\}$ and all edges incident with $u_{1}, u_{i}$ in an embedding of $H^{\prime}$ in $S_{1}$, we need face of minimum length 5 . Hence, for the embedding of $H^{\prime}$ in $S_{1}$, one of the following holds with respect to number of $k$-gons:

(1) $f_{3}=1, f_{4}=3, f_{5}=1$

(2) $f_{3}=2, f_{4}=1, f_{5}=2$

In each of the above two cases, there exists a face $f_{k}^{\prime}$ of $H^{\prime}$ of length 5 in $S_{1}$. First let us insert any one pair $\left\{\left(w_{j}, w_{j}^{\prime}\right): j=1,2,3\right\}$ and all the edges incident with $u_{1}, u_{i}$ into $f_{k}^{\prime}$ without any crossing as shown in Fig.4. In this case, the face $f_{k}^{\prime}$ is divided into 6 faces where each face has length less than 5 .

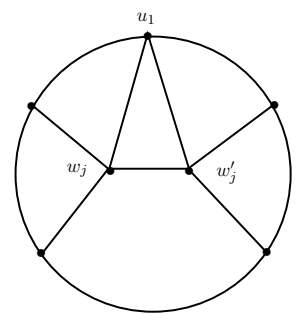

Fig. 4: The face $f_{k}^{\prime}$

Since face $f_{k}^{\prime}$ is divided into 6 faces with each faces has length less than 5 , no one can insert any more pair of vertices from $\left\{\left(w_{j}, w_{j}^{\prime}\right): j=1,2,3\right\}$ in $f_{k}^{\prime}$. Now consider the two cases mentioned above:

Case 1 Suppose $f_{3}=1, f_{4}=3$ and $f_{5}=1$. Since there exists a face has length 5 , as argued above, one can insert only one pair of vertices from $\left\{\left(w_{j}, w_{j}^{\prime}\right): j=1,2,3\right\}$ and other two pairs from $\left\{\left(w_{j}, w_{j}^{\prime}\right): j=1,2,3\right\}$ and all the edges incident with $u_{1}, u_{i}$ cannot be inserted into an embedding of $H^{\prime}$ in $S_{1}$.

Case 2 Suppose $f_{3}=2, f_{4}=1$ and $f_{5}=2$. Since there exist two faces of length 5 , so one 
can insert two pairs of vertices from $\left\{\left(w_{j}, w_{j}^{\prime}\right): j=1,2,3\right\}$ and all the edges incident with $u_{1}, u_{i}$ into embedding of $H^{\prime}$ in $S_{1}$. However one more remaining cannot be inserted into such embedding.

Hence in both the cases one cannot insert all pair of vertices $\left\{\left(w_{j}, w_{j}^{\prime}\right): j=1,2,3\right\}$ and incident edges of $H$ into any embedding on $S_{1}$. Hence we conclude that $g(H) \geq 2$.

Theorem 4.3. Let $n \geq 1$ and $q \geq 2$ be integers. Let $\mathbb{V}$ be an $n$ dimensional vector space over the field $\mathbb{F}$ with $q$ elements. Then $\Gamma\left(\mathbb{V}_{\mathfrak{B}}\right)$ is toroidal if and only if $n=1$ and $q=6,7,8$.

Proof. Let $\Gamma\left(\mathbb{V}_{\mathfrak{B}}\right)$ be toroidal. Suppose $n \geq 5$. Let $u_{1}=(1,1, \ldots, 1), u_{2}=(0,1, \ldots, 1)$, $u_{3}=(1,0,1, \ldots, 1), u_{4}=(1,1,0,1, \ldots, 1), u_{5}=(1,1,1,0,1, \ldots, 1)$ and $u_{6}=(1,1,1,1,0,1$, $\ldots, 1)$. Here the induced subgraph $H=\left\langle\left\{u_{1}, u_{2}, \ldots, u_{6}\right\}\right\rangle$ is $K_{6}$. Consider the toroidal embedding of $H$ given in Fig. 5 .

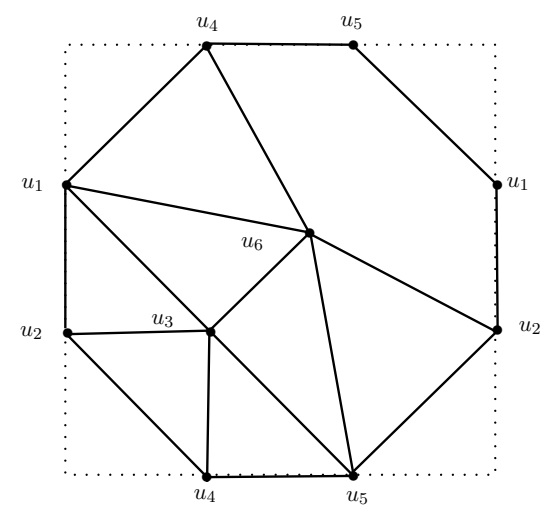

Fig. 5: Embedding $H$ in $S_{1}$

Let $u_{7}=(0,0,1,1,1,0,0, \ldots, 0), u_{8}=(1,0,1,1,0,0, \ldots, 0), u_{9}=(1,1,0,0,1,1, \ldots, 1)$ and $H^{\prime}=\left\langle\left\{u_{1}, u_{2}, \ldots, u_{9}\right\rangle\right\}$ be the induced subgraph. Note that $H \subset H^{\prime}$. Now, consider the toroidal embedding of $H^{\prime}-\left\{u_{9} u_{3}, u_{9} u_{4}\right\}$ given in the Fig. 6 .

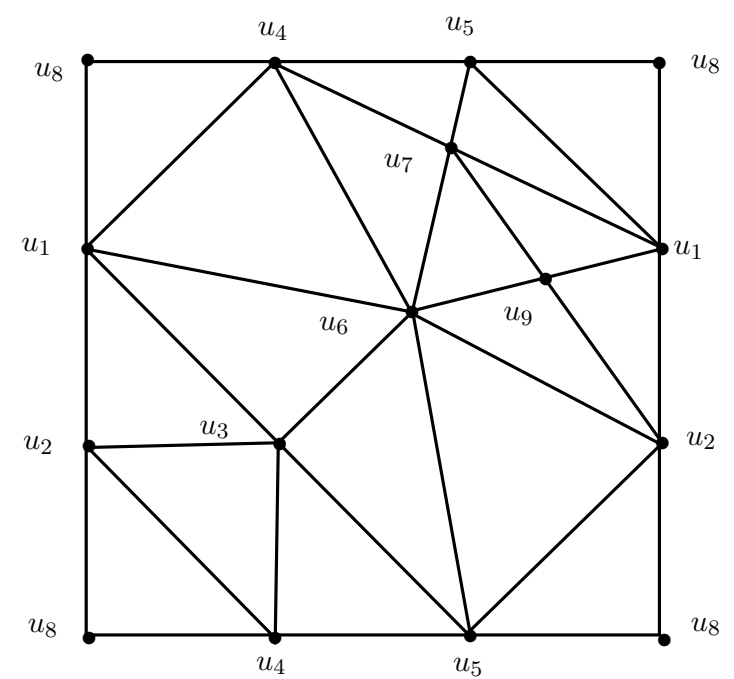

Fig. 6: Toroidal embedding of $H^{\prime}-\left\{u_{9} u_{3}, u_{9} u_{4}\right\}$

Since the Fig. 6 is triangulated, one cannot draw edges $u_{8} u_{2}$ and $u_{8} u_{3}$ without crossing, which is a contradiction. Hence $n \leq 4$.

Case 1. Let $n=4$.

Case 1.1 Suppose $q \geq 3$. Then $\mathbb{F}$ contains $a_{1}$ with $a_{1} \neq 0$ and $a_{1} \neq 1$. Consider the vertices $u_{1}=(1,1,1,1), u_{2}=(0,1,1,1), u_{3}=(1,0,1,1), u_{4}=(1,1,0,1), u_{5}=(1,1,1,0), u_{6}=$ 
$\left(a_{1}, 1,1,1\right), u_{7}=\left(1, a_{1}, 1,1\right)$, and $u_{8}=\left(1,1, a_{1}, 1\right)$. Note that the induced subgraph $\left\langle\left\{u_{1}, \ldots, u_{8}\right\}\right\rangle$ is $K_{8}$. By Lemma $2.7, g\left(\Gamma\left(\mathbb{V}_{\mathfrak{B}}\right)\right) \geq g\left(K_{8}\right) \geq 2$, which is a contradiction.

Case 1.2. Suppose $q=2$. Then $\mathbb{V} \cong \mathbb{Z}_{2} \times \mathbb{Z}_{2} \times \mathbb{Z}_{2} \times \mathbb{Z}_{2}$ and $\mathbb{F} \cong \mathbb{Z}_{2}$. Here $V\left(\Gamma\left(\mathbb{V}_{\mathfrak{B}}\right)\right)=$ $\left\{u_{1} \ldots, u_{15}\right\}$ where $u_{1}=(1,1,1,1), u_{2}=(0,1,1,1), u_{3}=(1,0,1,1), u_{4}=(1,1,0,1), u_{5}=$ $(1,1,1,0), \quad u_{6}=(1,1,0,0), u_{7}=(0,1,1,0), u_{8}=(0,0,1,1), u_{9}=(1,0,0,1), u_{10}=$ $(1,0,1,0), u_{11}=(0,1,0,1), u_{12}=(1,0,0,0), u_{13}=(0,1,0,0), u_{14}=(0,0,1,0), u_{15}=$ $(0,0,0,1)$.

Consider the mapping $\lambda$ defined by $\lambda\left(u_{i}\right)=u_{i}$ for $1 \leq i \leq 5, \lambda\left(u_{6}\right)=w_{1}, \lambda\left(u_{7}\right)=w_{2}$, $\lambda\left(u_{8}\right)=w_{1}^{\prime}, \lambda\left(u_{9}\right)=w_{2}^{\prime}, \lambda\left(u_{10}\right)=w_{3}$, and $\lambda\left(u_{11}\right)=w_{3}^{\prime}$. One can check that this mapping $\lambda$ induces an isomorphism between the induced subgraph $\left\langle\left\{u_{i}: 1 \leq i \leq 11\right\}\right\rangle$ of $\Gamma\left(\mathbb{V}_{\mathfrak{B}}\right)$ and $H$ given in Fig. 3. By Lemma 4.2, $g\left(\Gamma\left(\mathbb{V}_{\mathfrak{B}}\right)\right) \geq g(H)=2$, which is a contradiction.

Case 2. Let $n=3$.

Case 2.1. Suppose $q \geq 3$. Then $\mathbb{F}$ contains $a_{1}$ with $a_{1} \neq 0$, and $a_{1} \neq 1$. Let $u_{1}=(1,1,1), u_{2}=\left(a_{1}, a_{1}, a_{1}\right), u_{3}=\left(a_{1}, 1,1\right), u_{4}=\left(1, a_{1}, 1\right), u_{5}=\left(1,1, a_{1}\right), u_{6}=$ $\left(a_{1}, a_{1}, 1\right), u_{7}=\left(1, a_{1}, a_{1}\right), u_{8}=\left(a_{1}, 1, a_{1}\right)$. Then the induced subgraph $\left\langle\left\{u_{1}, \ldots, u_{8}\right\}\right\rangle$ of $\Gamma\left(\mathbb{V}_{\mathfrak{B}}\right)$ is $K_{8}$. By Lemma 2.7, $g\left(\Gamma\left(\mathbb{V}_{\mathfrak{B}}\right)\right) \geq g\left(K_{8}\right) \geq 2$, which is a contradiction.

Case 2.1. Suppose $q=2$. By Theorem 4.1, $\Gamma\left(\mathbb{V}_{\mathfrak{B}}\right)$ is planar, which is a contradiction.

Case 3. Let $n=2$.

Case 3.1. Suppose $q \geq 3$. Then $\mathbb{F}$ contains $a_{1}$ with $a_{1} \neq 0$, and $a_{1} \neq 1$. Let $u_{1}=$ $(1,1), u_{2}=\left(a_{1}, a_{1}\right), u_{3}=\left(a_{1}, 1\right), u_{4}=\left(1, a_{1}\right), u_{5}=(1,0), u_{6}=(0,1), u_{7}=\left(a_{1}, 0\right)$. Note that the subgraph $H$ of $\Gamma\left(\mathbb{V}_{\mathfrak{B}}\right)$ induced by $\left\{u_{1}, \ldots, u_{7}\right\}$ is $K_{7}-u_{5} u_{7}$. Here $H$ has 7 vertices and 20 edges. Consider the toroidal embedding of $H$ given in the Fig. 7 .

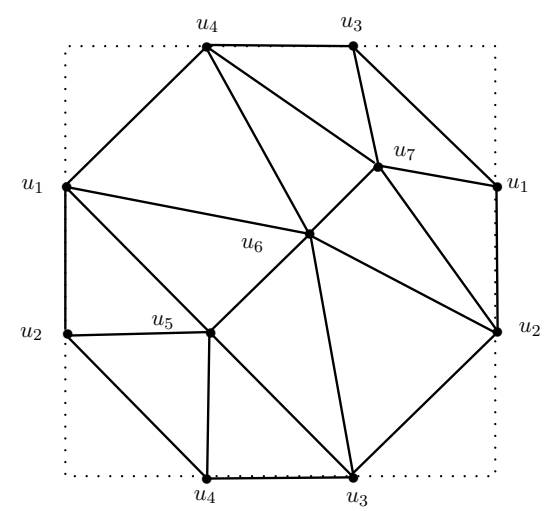

Fig. 7: Toroidal embedding of $H$

Applying Lemma 2.8, we get that $H$ has 13 faces. Let $f_{k}$ be the number of $k$-gons in $H$. Then $40=2 e=\sum_{k \geq 3} k f_{k}$. Hence, an embedding with 13 faces of $H$ in $S_{1}$ is possible only when $f_{3}=12$ and $f_{4}=1$.

By Theorem 2.5, degree of $\left(0, a_{1}\right)$ is at least 6 and so one cannot insert the vertex $u_{8}=\left(0, a_{1}\right)$ and all the edges incident with $u_{8}$ in Fig. 7 without crossing the edges. Therefore $g\left(\Gamma\left(\mathbb{V}_{\mathfrak{B}}\right)\right) \geq g(H) \geq 2$, which is a contradiction.

Case 3.2. Suppose $q=2$. By Theorem 4.1, $\Gamma\left(\mathbb{V}_{\mathfrak{B}}\right)$ is planar, which is a contradiction.

Case 4. Let $n=1$.

Case 4.1. Suppose $q \geq 9$. By Theorem 2.1 and $2.4, \Gamma\left(\mathbb{V}_{\mathfrak{B}}\right)$ is not toroidal, a contradiction to the assumption.

Case 4.2. Suppose $q \leq 5$. By Theorem $4.1, \Gamma\left(\mathbb{V}_{\mathfrak{B}}\right)$ is planar which is a contradiction.

Hence $n=1$ and $q=6,7,8$.

Conversely follows from Theorems 2.1 and 2.7.

Now, we characterize all finite dimensional vector space $\mathbb{V}$ over a finite field $\mathbb{F}$ for which $\Gamma\left(\mathbb{V}_{\mathfrak{B}}\right)$ is of genus two. 
Theorem 4.4. Let $n \geq 1$ and $q \geq 2$ be integers. Let $\mathbb{V}$ be a $n$-dimensional vector space over the field $\mathbb{F}$ with $q$ elements. Then $\Gamma\left(\mathbb{V}_{\mathfrak{B}}\right)$ is of genus two if and only if either $(n=1$ and $q=9)$ or $(n=2$ and $q=3)$ or $(n=4$ and $q=2)$.

Proof. Assume that $g\left(\Gamma\left(\mathbb{V}_{\mathfrak{B}}\right)\right)=2$. Suppose $n \geq 5$.

Let $u_{1}=(1,1, \ldots, 1), u_{2}=(0,1, \ldots, 1), u_{3}=(1,0,1, \ldots, 1), u_{4}=(1,1,0,1, \ldots, 1), u_{5}=$ $(1,1,1,0,1, \ldots, 1), u_{6}=(1,1,1,1,0,1, \ldots, 1), u_{7}=(0,1,0,1,0,1,1, \ldots, 1), u_{8}=(1,0,1,0$, $1,1, \ldots, 1), u_{9}=(1,0,1,1,0,1,1, \ldots, 1), u_{10}=(0,0,1,1, \ldots, 1), u_{11}=(0,1,0,1,1, \ldots, 1)$, $u_{12}=(1,1,1,0,0,1,1, \ldots, 1)$ and $u_{13}=(1,1,0,0,1,1, \ldots, 1)$. Then the subgraph $H$ of $\Gamma\left(\mathbb{V}_{\mathfrak{B}}\right)$ induced by $\Omega=\left\{u_{i}: 1 \leq i \leq 13\right\}$ is given in the Fig. 8 .

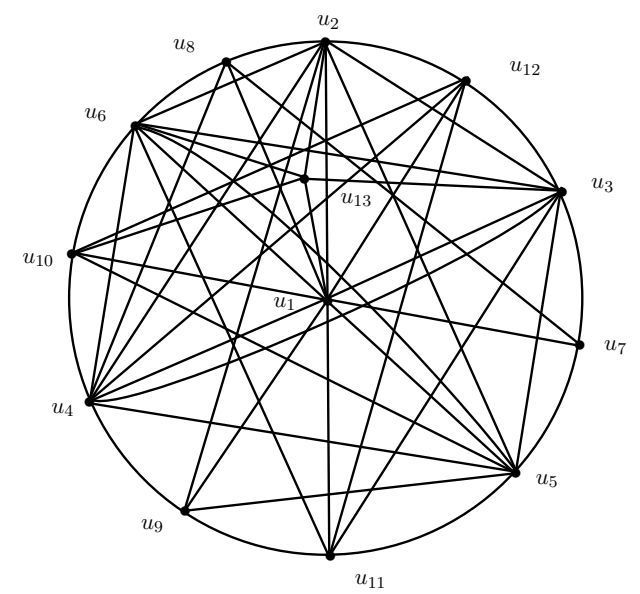

Fig. 8: Graph $H$

Now, consider the subgraph $H^{\prime}=H-u_{13}$ of $H$ with 12 vertices and 42 edges. By Lemma $2.8, H^{\prime}$ has 28 faces. Let $f_{k}$ be the number of $k$-gons in $H^{\prime}$. Then $84=2 e=\sum_{k \geq 3} k f_{k}$. Hence an embedding of $H^{\prime}$ with 28 faces in $S_{2}$ is possible only when $f_{3}=28$.

Suppose there exists an embedding of $H^{\prime}$ on $S_{2}$ which is triangulated. Since the degree of $u_{13}$ is 5 , one cannot insert the vertex $u_{13}$ and all edges adjacent with vertices in $H^{\prime}$ without crossing the edges on $S_{2}$. Therefore $g\left(\Gamma\left(\mathbb{V}_{\mathfrak{B}}\right)\right) \geq g(H) \geq 3$, which is a contradiction. Hence $n \leq 4$.

Case 1. Let $n=4$. Suppose $q \geq 3$. Then $\mathbb{F}$ contains $a_{1}$ with $a_{1} \neq 0$, and $a_{1} \neq 1$. Let $u_{1}=(1,1,1,1), u_{2}=(0,1,1,1), u_{3}=(1,0,1,1), u_{4}=(1,1,0,1), u_{5}=(1,1,1,0), u_{6}=$ $\left(a_{1}, 1,1,1\right), u_{7}=\left(1, a_{1}, 1,1\right), u_{8}=\left(1,1, a_{1}, 1\right)$ and $u_{9}=\left(1,1,1, a_{1}\right)$. Then the induced subgraph $\left\langle\left\{u_{1}, u_{2}, \ldots, u_{9}\right\}\right\rangle$ is $K_{9}$. By Lemma 2.7, $g\left(\Gamma\left(\mathbb{V}_{\mathfrak{B}}\right)\right) \geq 3$, which is a contradiction.

Hence $n=4$ and $q=2$.

Case 2. Let $n=3$.

Case 2.1. Suppose $q \geq 3$. Then $\mathbb{F}$ contains $a_{1}$ with $a_{1} \neq 0$, and $a_{1} \neq 1$. Let $u_{1}=(1,1,1), u_{2}=(0,1,1), u_{3}=\left(a_{1}, a_{1}, a_{1}\right), u_{4}=\left(a_{1}, 1,1\right), u_{5}=\left(1, a_{1}, 1\right), u_{6}=$ $\left(1,1, a_{1}\right), u_{7}=\left(a_{1}, a_{1}, 1\right), u_{8}=\left(1, a_{1}, a_{1}\right), u_{9}=\left(a_{1}, 1, a_{1}\right)$. Then the induced subgraph $\left\langle\left\{u_{1}, u_{2}, \ldots, u_{9}\right\}\right\rangle$ is $K_{9}$ and is a subgraph in $\Gamma\left(\mathbb{V}_{\mathfrak{B}}\right)$. By Lemma 2.7, $g(H)=3$ and so $g\left(\Gamma\left(\mathbb{V}_{\mathfrak{B}}\right)\right) \geq 3$, which is a contradiction.

Case 2.2. Suppose $q=2$. By Theorem $4.1, \Gamma\left(\mathbb{V}_{\mathfrak{B}}\right)$ is planar, which is a contradiction to our assumption that $\Gamma\left(\mathbb{V}_{\mathfrak{B}}\right)$ is of genus two. Therefore $n \neq 3$.

Case 3. Let $n=2$.

Case 3.1. Suppose $q=2$. By Theorem $4.1, \Gamma\left(\mathbb{V}_{\mathfrak{B}}\right)$ is planar, which is a contradiction.

Case 3.2. Suppose $q \geq 4$. Then $\mathbb{F}$ contains $a_{1}, a_{2}$ with $a_{1}, a_{2} \neq 0$ and $a_{1}, a_{2} \neq 1$. Let $u_{1}=(1,1), u_{2}=(0,1), u_{3}=(0,1), u_{4}=\left(a_{2}, a_{2}\right), u_{5}=\left(1, a_{1}\right), u_{6}=\left(1, a_{2}\right), u_{7}=$ $\left(a_{1}, 1\right), u_{8}=\left(a_{2}, 1\right)$, and $u_{9}=\left(a_{1}, a_{1}\right)$. Note that the induced subgraph $\left\langle\left\{u_{1}, \ldots, u_{9}\right\}\right\rangle$ is $K_{9}$. By Lemma $2.4, g\left(\Gamma\left(\mathbb{V}_{\mathfrak{B}}\right)\right) \geq g\left(K_{9}\right) \geq 3$, which is a contradiction. 
From the above, we have that $n=2$ and $q=3$.

Case 4. Let $n=1$. Suppose $q \leq 5$. By Theorem 4.1, $g\left(\Gamma\left(\mathbb{V}_{\mathfrak{B}}\right)\right)=0$, a contradiction. Suppose $6 \leq q \leq 8$. By Theorem 4.3, $g\left(\Gamma\left(\mathbb{V}_{\mathfrak{B}}\right)\right)=1$, a contradiction. Hence $q \geq 9$. Suppose $q \geq 10$. By Theorem 2.1, $\Gamma\left(\mathbb{V}_{\mathfrak{B}}\right) \cong K_{9}$. By Lemma 2.7, $g\left(\Gamma\left(\mathbb{V}_{\mathfrak{B}}\right)\right)=3$, which is a contradiction to the fact that $\Gamma\left(\mathbb{V}_{\mathfrak{B}}\right)$ is of genus two.

Hence $n=1$ and $q=9$.

Conversely, assume that $n=1$ and $q=9$. Then $\Gamma\left(\mathbb{V}_{\mathfrak{B}}\right) \cong K_{8}$. By Lemma 2.4, $g\left(\Gamma\left(\mathbb{V}_{\mathfrak{B}}\right)\right)=2$.

Assume that $n=2$ and $q=3$. Then $\mathbb{V} \cong \mathbb{Z}_{3} \times \mathbb{Z}_{3}$ and $\mathbb{F}=\mathbb{Z}_{3}$. By Theorem 2.4, order of $\Gamma\left(\mathbb{V}_{\mathfrak{B}}\right)$ is 8 and so $\Gamma\left(\mathbb{V}_{\mathfrak{B}}\right) \subseteq K_{8}$. By Lemma 2.7, $g\left(\Gamma\left(\mathbb{V}_{\mathfrak{B}}\right)\right) \leq 2$. On the other hand, by Theorem 4.3, $g\left(\Gamma\left(\mathbb{V}_{\mathfrak{B}}\right)\right)>1$. Therefore $g\left(\Gamma\left(\mathbb{V}_{\mathfrak{B}}\right)\right)=2$.

Assume that $n=4$ and $q=2$. Then $\mathbb{V} \cong \mathbb{Z}_{2} \times \mathbb{Z}_{2} \times \mathbb{Z}_{2} \times \mathbb{Z}_{2}$ and $\mathbb{F}=\mathbb{Z}_{2}$. An embedding of $\Gamma\left(\mathbb{V}_{\mathfrak{B}}\right)$ in $S_{2}$ is given in the Fig. 9. Hence $g\left(\Gamma\left(\mathbb{V}_{\mathfrak{B}}\right)\right)=2$.

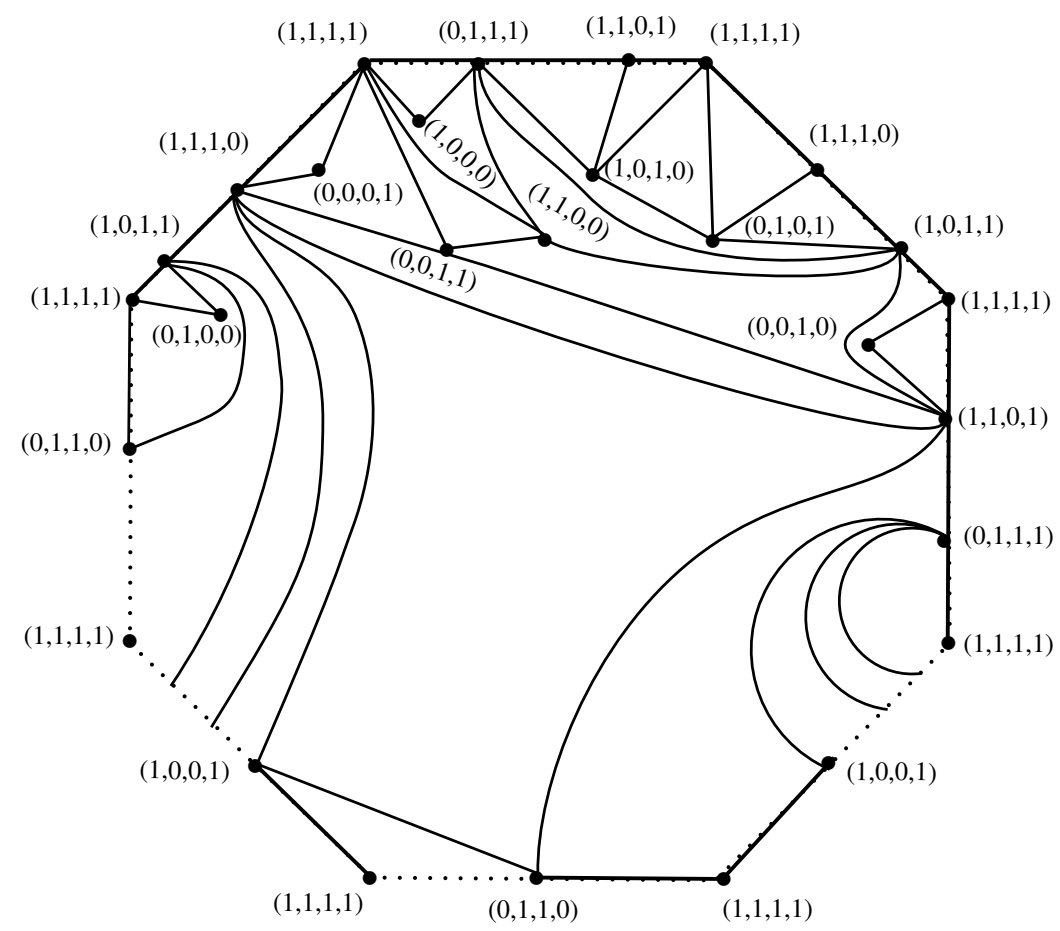

Fig. 9: An embedding of $\Gamma\left(\mathbb{Z}_{2} \times \mathbb{Z}_{2} \times \mathbb{Z}_{2} \times \mathbb{Z}_{2}\right)$ where $\mathbb{F}=\mathbb{Z}_{2}$

\section{Outerplanar property of $\Gamma\left(\mathbb{V}_{\mathfrak{B}}\right)$}

In this section, we characterize outerplanar and outerplanarity index of $\Gamma\left(\mathbb{V}_{\mathfrak{B}}\right)$ where $\mathbb{V}$ is a finite dimensional vector space. A graph is said to be outerplanar if it can be drawn in the plane without crossings in such a way that all of the vertices belong to the unbounded face of the drawing. Recall that, a graph $G$ is $k$-outerplanar if $G$ can be drawn in the plane without crossings such that after $k$-fold removal of the vertices on the outer-face, there are no vertices left. The minimum value of $k$ is called outerplanarity index. Inner vertex number $i(G)$ of a plane graph is defined as minimum number of vertices which are not in the exterior face of the planar embedding of $G$.

Theorem 5.1. Let $n \geq 1$ and $q \geq 2$ be integers. Let $\mathbb{V}$ be a $n$-dimensional vector space over the field $\mathbb{F}$ with $q$ elements. Then $\Gamma\left(\mathbb{V}_{\mathfrak{B}}\right)$ is outerplanar if and only if either $(n=1$ and $q \leq 4)$ or $(n=2$ and $q=2)$.

Proof. Assume that $\Gamma\left(\mathbb{V}_{\mathfrak{B}}\right)$ is outerplanar.

Suppose $n \geq 3$. Let $u_{1}=(1,1, \ldots, 1), u_{2}=(0,1,1, \ldots, 1), u_{3}=(1,0,1,1, \ldots, 1)$ and $u_{4}=(1,1,0,1,1, \ldots, 1)$. Note that the subgraph of $\Gamma\left(\mathbb{V}_{\mathfrak{B}}\right)$ induced by $\left\{u_{1}, u_{2}, u_{3}, u_{4}\right\}$ is $K_{4}$. 
By Theorem 2.11, $K_{4}$ is not outerplanar and so $\Gamma\left(\mathbb{V}_{\mathfrak{B}}\right)$ is not outerplanar, a contradiction. Hence $n \leq 2$.

Case 1. Assume $n=1$. Suppose $q \geq 5$. By Theorem 2.1, $\Gamma\left(\mathbb{V}_{\mathfrak{B}}\right) \cong K_{4}$. By Theorem 2.11, $\Gamma\left(\mathbb{V}_{\mathfrak{B}}\right)$ is not a outerplanar, a contradiction. Therefore $q \leq 4$.

Hence $n=1$ and $q \leq 4$.

Case 2. Assume $n=2$. Suppose $q \geq 3$. Then $\mathbb{F}$ contains $a_{1}$ with $a_{1} \neq 0$ and $a_{1} \neq$ 1. Let $u_{1}=(1,1), u_{2}=(0,1), u_{3}=(1,0), u_{4}=\left(a_{1}, a_{1}\right)$. Note that the subgraph induced by $\left\{u_{1}, u_{2}, u_{3}, u_{4}\right\}$ is $K_{4} \subset \Gamma\left(\mathbb{V}_{\mathfrak{B}}\right)$. By Theorem $2.11, \Gamma\left(\mathbb{V}_{\mathfrak{B}}\right)$ is not a outerplanar, a contradiction Therefore $q=2$.

Conversely, assume that $n=1$ and $q=2,3,4$. By Theorem $2.1, \Gamma\left(\mathbb{V}_{\mathfrak{B}}\right) \cong K_{1}, K_{2}$, and $K_{3}$ respectively. It is obvious that $K_{1}, K_{2}$ and $K_{3}$ are outerplanar.

Suppose $n=2$ and $q=2$. Then by Theorem 2.1, $\Gamma\left(\mathbb{V}_{\mathfrak{B}}\right) \cong K_{3}$, which is outerplanar.

By Theorems 4.1 and 5.1, we have the following corollary.

Corollary 5.2. Let $n \geq 1$ and $q \geq 2$ be integers. Let $\mathbb{V}$ be a $n$-dimensional vector space over the field $\mathbb{F}$ with $q$ elements. Then $\Gamma\left(\mathbb{V}_{\mathfrak{B}}\right)$ is planar but not outerplanar if and only if either $(n=1$ and $q=5)$ or $(n=3$ and $q=2)$.

Theorem 5.3. Let $n \geq 1$ and $q \geq 2$ be integers. Let $\mathbb{V}$ be a $n$-dimensional vector space over the field $\mathbb{F}$ with $q$ elements. Then the outerplanarity index of $\Gamma\left(\mathbb{V}_{\mathfrak{B}}\right)$ is either 1 or 2.

Proof. By the Theorem 5.1, $\Gamma\left(\mathbb{V}_{\mathfrak{B}}\right)$ is outerplanar (1-outerplanar) if and only if either $(n=1$ and $q \leq 4)$ or $(n=2$ and $q=2)$. By Corollary $5.2, \Gamma\left(\mathbb{V}_{\mathfrak{B}}\right)$ is planar and not outerplanar if and only if either $(n=1$ and $q=5)$ or $(n=3$ and $q=2$.).

Case 1. Suppose $n=1$ and $q=5$. By Theorem $2.1, \Gamma\left(\mathbb{V}_{\mathfrak{B}}\right) \cong K_{4}$. Note that $K_{4}$ is 2-outerplanar graph and so the outerplanar index of $\Gamma\left(\mathbb{V}_{\mathfrak{B}}\right)$ is 2 .

Case 2. Suppose $n=3$ and $q=2$. Then the planar embedding of $\Gamma\left(\mathbb{V}_{\mathfrak{B}}\right)$ is given in the Fig. 2. Removing the three vertices $\{(1,1,0),(1,0,1),(0,1,1)\}$ in the unbounded face, we get the star graph which is outerplanar. Hence in this case outerplanar index of $\Gamma\left(\mathbb{V}_{\mathfrak{B}}\right)$ is 1.

Therefore outerplanarity index of $\Gamma\left(\mathbb{V}_{\mathfrak{B}}\right)$ is either 1 or 2 .

Theorem 5.4. Let $n \geq 1$ and $q \geq 2$ be integers. Let $\mathbb{V}$ be a $n$-dimensional vector space over the field $\mathbb{F}$ with $q$ elements. Then inner vertex number of $\Gamma\left(\mathbb{V}_{\mathfrak{B}}\right)$ is given by

$$
i\left(\Gamma\left(\mathbb{V}_{\mathfrak{B}}\right)\right)= \begin{cases}0, & \text { if }(n=1 \text { and } q \leq 4) \text { or }(n=2 \text { and } q=2) \\ 1, & \text { if } n=1 \text { and } q=5 \\ 2, & \text { if } n=3 \text { and } q=2 .\end{cases}
$$

Proof. Case 1. Let $(n=1$ and $q \leq 4)$ or $(n=2$ and $q=2)$. By Theorem $5.1, \Gamma\left(\mathbb{V}_{\mathfrak{B}}\right)$ is outerplanar so it is a 1-outerplanar graph. Hence $i\left(\Gamma\left(\mathbb{V}_{\mathfrak{B}}\right)\right)=0$.

Case 2. Let $n=1$ and $q=5$. By Theorem $2.1, \Gamma\left(\mathbb{V}_{\mathfrak{B}}\right) \cong K_{4}$. By Theorem $5.3 \Gamma\left(\mathbb{V}_{\mathfrak{B}}\right)$ is 2-outerplanar. Since the minimum number of vertices in an interior face is one, so $i\left(\Gamma\left(\mathbb{V}_{\mathfrak{B}}\right)\right)=1$.

Case 3. Let $n=3$ and $q=2$. Then $\mathbb{V} \cong \mathbb{Z}_{2} \times \mathbb{Z}_{2} \times \mathbb{Z}_{2}$ and $\mathbb{F}=\mathbb{Z}_{2}$. A planar embedding of $\Gamma\left(\mathbb{V}_{\mathfrak{B}}\right)$ is given by the Fig. 10(b). Note that, the graph $H$ given in Fig. 10(a) is a maximal outer planar subgraph of $\Gamma\left(\mathbb{V}_{\mathfrak{B}}\right)$. If we insert the edges $((1,1,1)(1,1,0)),((1,1,1)(0,0,1))$ and $((0,0,1)(1,1,0))$, then we get two as the minimum inner vertex. Hence $i\left(\Gamma\left(\mathbb{V}_{\mathfrak{B}}\right)\right)=$ 2 . 


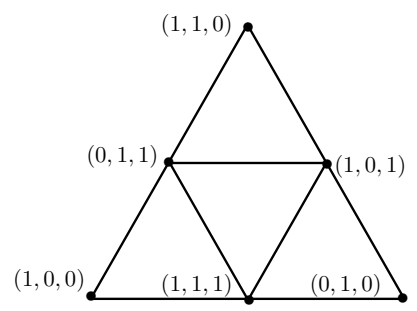

Fig. 10(a) : A subgraph $H$ of $\Gamma\left(\mathbb{Z}_{2} \times \mathbb{Z}_{2} \times \mathbb{Z}_{2}\right)$

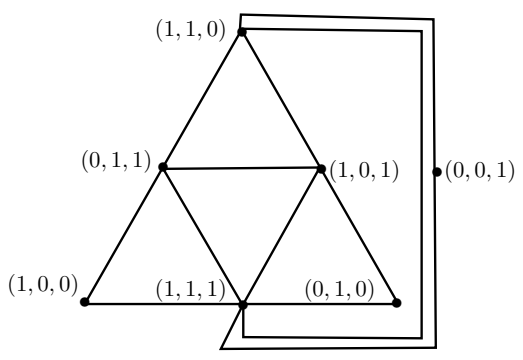

Fig. 10(b) : $\Gamma\left(\mathbb{Z}_{2} \times \mathbb{Z}_{2} \times \mathbb{Z}_{2}\right)$ with $\mathbb{F}=\mathbb{Z}_{2}$

\section{Crosscap of $\Gamma\left(\mathbb{V}_{\mathfrak{B}}\right)$}

In this section, we characterize all finite dimensional vector spaces whose non-zero component union graph is of crosscap one.

Lemma 6.1. Let $H$ be a graph shown in Fig. 3. Then $\bar{g}(H) \geq 2$.

Proof. Note that the induced subgraph $H^{\prime}=\left\langle\left\{u_{1}, u_{2}, u_{3}, u_{4}, u_{5}\right\}\right\rangle$ of $H$ is $K_{5}$. By Theorem $2.9, \bar{g}\left(H^{\prime}\right)=1$. Note that $H^{\prime}$ has 5 vertices and 10 edges. Let $f_{k}$ be the number of $i$-gons in $H^{\prime}$. Then $20=2 e=\sum_{k \geq 3} k f_{k}$.

By Proposition 2.10, any embedding of $H^{\prime}$ has 6 faces in $N_{1}$.

Note that $\left\{w_{1}, w_{1}^{\prime}\right\},\left\{w_{2}, w_{2}^{\prime}\right\}$ and $\left\{w_{3}, w_{3}^{\prime}\right\}$ are pair of adjacent vertices with one common neighbourhood $u_{1}$. Also, every vertex in each of these pairs, has two distinct adjacent neighbourhoods in $\left\{u_{i}: i=2,3,4,5\right\}$. To insert one pair from $\left\{w_{j}, w_{j}^{\prime}: j=1,2,3\right\}$ and all edges incident with $u_{1}, u_{i}, w_{j}, w_{j}^{\prime}$, then we need a face of minimum length 5 .

Thus an embedding of $H^{\prime}$ in $N_{1}$ needs either of the following:

(1) $f_{3}=5, f_{5}=1$;

(2) $f_{3}=4, f_{4}=2$.

Suppose there exists a face $f^{\prime}$ of length 5 . After inserting one pair from $\left\{\left(w_{j}, w_{j}^{\prime}\right): j=\right.$ $1,2,3\}$ and all edges incident with $u_{1}, u_{i}, w_{j}, w_{j}^{\prime}$ in $f^{\prime}$, we get a graph $F$ given in Fig. 11 .

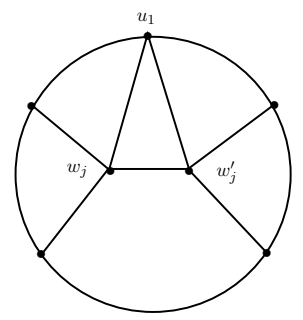

Fig. 11: The graph $F$ and face $f^{\prime}$

Since each face has length less than 5 , no one can insert any more pair of vertices from $\left\{\left(w_{j}, w_{j}^{\prime}\right): j=1,2,3\right\}$ and all incident edges in $f^{\prime}$. Now consider the two cases mentioned above:

Case 1. Suppose $f_{3}=5$ and $f_{5}=1$. Since there exists a face has length 5 , as argued above, one can insert only one pair of vertices from $\left\{\left(w_{j}, w_{j}^{\prime}\right): j=1,2,3\right\}$ and other two pairs from $\left\{\left(w_{j}, w_{j}^{\prime}\right): j=1,2,3\right\}$ and all the edges incident with $u_{1}, u_{i}$ cannot be inserted into an embedding of $H^{\prime}$ in $N_{1}$.

Case 2 Suppose $f_{3}=4, f_{4}=2$. There is no face of length greater than or equal to 5 , no one can insert any pair of vertices from $\left\{\left(w_{j}, w_{j}^{\prime}\right): j=1,2,3\right\}$ and all the edges incident with $u_{1}, u_{i}$ into an embedding of $H^{\prime}$ in $N_{1}$.

Hence in both the cases one cannot insert all pair of vertices $\left\{\left(w_{j}, w_{j}^{\prime}\right): j=1,2,3\right\}$ and incident edges of $H$ into any embedding on $N_{1}$. Hence we conclude that $\bar{g}(H) \geq 2$. 
Theorem 6.2. Let $n \geq 1$ and $q \geq 2$ be integers. Let $\mathbb{V}$ be a $n$-dimensional vector space over the field $\mathbb{F}$ with $q$ elements. Then $\bar{g}\left(\Gamma\left(\mathbb{V}_{\mathfrak{B}}\right)\right)=1$ if and only if $n=1$ and $q=6$ or 7 .

Proof. Assume that $\bar{g}\left(\left(\Gamma\left(\mathbb{V}_{\mathfrak{B}}\right)\right)=1\right.$. Suppose $n \geq 4$. Let $u_{1}=(1,1, \ldots, 1), u_{2}=(0,1,1$, $\ldots, 1), u_{3}=(1,0,1,1, \ldots, 1), u_{4}=(1,1,0,1,1, \ldots, 1), u_{5}=(1,1,1,0,1,1, \ldots, 1), u_{6}=$ $(1,1,0,0,1,1, \ldots, 1), u_{7}=(0,1,1,0,1,1, \ldots, 1), u_{8}=(0,0,1,1, \ldots, 1), u_{9}=(1,0,0,1,1$, $\ldots, 1), u_{10}=(1,0,1,0,1,1, \ldots, 1)$, and $u_{11}=(0,1,0,1,1, \ldots, 1)$. Then the induced subgraph $\left\langle\left\{u_{1}, \ldots, u_{11}\right\}\right\rangle$ is isomorphic to $H$ in Fig. 3. Applying Lemma 6.1, we get that $\bar{g}\left(\left(\Gamma\left(\mathbb{V}_{\mathfrak{B}}\right)\right) \geq \bar{g}(H) \geq 2\right.$, which is a contradiction. Hence $n \leq 3$.

Case 1. Let $n=3$. If $q=2$, then by Theorem $4.1 \Gamma\left(\mathbb{V}_{\mathfrak{B}}\right)$ is planar, a contradiction. If $q \geq 3$, then by Theorems 2.2 and $2.9, \bar{g}(H) \geq 10$, a contradiction.

Case 2. Let $n=2$. If $q=2$, then by Theorem 4.1, $\Gamma\left(\mathbb{V}_{\mathfrak{B}}\right)$ is planar a contradiction. If $q \geq 3$, then by Theorem 2.2, clique number of $\Gamma\left(\mathbb{V}_{\mathfrak{B}}\right)$ is at least 6 and so $H=K_{6} \subseteq \Gamma\left(\mathbb{V}_{\mathfrak{B}}\right)$. By Theorem 2.10, $H$ in $N_{1}$ is triangulated. By Theorem 2.4, order of $\Gamma\left(\mathbb{V}_{\mathfrak{B}}\right)$ is 8 and by Theorem 2.5, minimum degree of $\Gamma\left(\mathbb{V}_{\mathfrak{B}}\right)$ is 6 . This shows that existence of at least one vertex $u$ not in $H$ with degree greater than or equal to 6 . One cannot insert $u$ and all incident edges in the embedding of $H$ on $N_{1}$ and so $\bar{g}\left(\Gamma\left(\mathbb{V}_{\mathfrak{B}}\right)\right) \geq \bar{g}(H) \geq 2$, a contradiction.

Case 3. Let $n=1$. If $q \leq 5$, by Theorem $4.1, \Gamma\left(\mathbb{V}_{\mathfrak{B}}\right)$ is planar, a contradiction. If $q \geq 8$, then by Theorem 2.1 and Theorem $2.9, \bar{g}\left(\Gamma\left(\mathbb{V}_{\mathfrak{B}}\right)\right)=3$, which is a contradiction. Hence, only possible values of $q$ are 6 or 7 .

Thus $n=1$ and $q=6$ or 7 .

Conversely, assume that $n=1$ and $q=6,7$. By Theorem 2.1 and 2.9, $\bar{g}\left(\Gamma\left(\mathbb{V}_{\mathfrak{B}}\right)\right)=$ 1.

Acknowledgment. The research of T. Tamizh Chelvam is supported by CSIR Emeritus Scientist Scheme (No.21 (1123)/ 20/ EMR-II) of Council of Scientific and Industrial Research, Government of India.

\section{References}

[1] D.F. Anderson and P.S. Livingston, The zero-divisor graph of a commutative ring, J. Algebra, 217, 434-447, 1999.

[2] I. Beck, Coloring of commutative rings, J. Algebra, 116, 208-226, 1988.

[3] J.A. Bondy and U.S.R. Murty, Graph Theory with Applications, Elsevier, North Holland, Amsterdam, 1986.

[4] A. Das, Non-zero component union graph of a finite-dimensional vector space, Linear Multilinear Algebra, 65(6), 1276-1287, 2016.

[5] A. Das, Nonzero Component graph of a finite dimensional vector space, Comm. Algebra, 44(9), 3918-3926, 2016.

[6] G. Kalaimurugan, P. Vignesh and T. Tamizh Chelvam, On zero divisor graphs of commutative rings without identity, J. Algebra Appl. 19(12), \# 2050226, 2020.

[7] B. Mohar and C. Thomassen, Graphs on Surfaces, Johns Hopkins University Press, 2001.

[8] K. Selvakumar and P. Subbulakshmi, On the crosscap of the annihilating-ideal graph of a commutative ring, Palest. J. Math. 7(1), 151-160, 2018.

[9] M. Syszo, Characterizations of outerplanar graphs, Discrete Math. 26, 47-53, 1979.

[10] T. Tamizh Chelvam and T. Asir, Genus of total graphs from rings: A survey, AKCE Int. J. Graphs Comb. 15(1), 97-104, 2018.

[11] T. Tamizh Chelvam and S. Nithya, Crosscap of the ideal based zero-divisor graph, Arab J. Math. Sci. 22, 29-37, 2016.

[12] T. Tamizh Chelvam and K. Prabha Ananthi, On the genus of graphs associated with vector spaces, J. Algebra Appl. 5, \# 2050086, 2019. 
[13] T. Tamizh Chelvam and K. Prabha Ananthi, Complement of the reduced nonzero component graph of free semi-modules, Accepted for publication in Applied Mathematics-A Journal of Chinese Universities.

[14] T. Tamizh Chelvam and K. Selvakumar, On the genus of the annihilator graph of a commutative ring, Algebra Discrete Math. 24(2), 191-208, 2017.

[15] A.T. White, Graphs, Groups and Surfaces, North-Holland, Amsterdam, 1973. 